\title{
Lymph node density is a prognostic factor in patients with major salivary gland carcinoma
}

\author{
HIDENORI SUZUKI, NOBUHIRO HANAI, HITOSHI HIRAKAWA, \\ DAISUKE NISHIKAWA and YASUHISA HASEGAWA
}

Department of Head and Neck Surgery, Aichi Cancer Center Hospital, Nagoya, Aichi 464-8681, Japan

Received November 11, 2014; Accepted September 18, 2015

DOI: $10.3892 / \mathrm{ol} .2015 .3814$

\begin{abstract}
Lymph node density (LND) has been reported to be a significant predictor of survival in patients with oral and other carcinomas exhibiting positive lymph nodes. The present study investigated whether the LND is associated with overall survival in subjects with major salivary gland carcinoma. A total of 78 patients newly diagnosed with major salivary gland carcinoma underwent primary tumor resection and neck dissection without preoperative treatment. Of these 78 patients, 32 with pathologically positive lymph nodes were enrolled in the present study. The LND was calculated as the ratio of the number of positive lymph nodes to the number of total lymph nodes. The survival rate was analyzed according to the Kaplan-Meier method. A univariate survival analysis was performed using the log-rank test, and a multivariate survival analysis was performed using the Cox proportional hazards model. An LND of $\geq 0.38$ was found to significantly correlate with a shorter overall survival time in univariate analysis $(\mathrm{P}=0.017)$. In multivariate survival analysis, after adjusting for anatomical location (parotid gland/others), an LND of $\geq 0.38$ was identified to be associated with a significantly shorter overall survival time. These results suggest that the LND functions as a prognostic factor in cases of major salivary gland carcinoma.
\end{abstract}

\section{Introduction}

Major salivary gland carcinomas, which are distinct from parotid gland carcinomas, submandibular gland carcinomas and sublingual gland carcinomas, are rare malignant tumors, accounting for $<5 \%$ of all cancers of the head and neck (1). The histological classification of major salivary gland carcinomas comprises 24 histological subtypes with different malignant

Correspondence to: Dr Hidenori Suzuki, Department of Head and Neck Surgery, Aichi Cancer Center Hospital, 1-1 Kanokoden, Chikusa-ku, Nagoya, Aichi 464-8681, Japan

E-mail: hi.suzuki@aichi-cc.jp

Key words: lymph node density, major salivary gland carcinoma, overall survival phenotypes and prognoses, according to the 2005 World Health Organization classification of tumors (2). To date, numerous studies have investigated the characteristics and useful prognostic parameters of major salivary gland carcinoma using various approaches, including clinical, pathological and biological procedures (1-4).

The lymph node density (LND), which is calculated as the ratio of the number of positive lymph nodes to the number of total lymph nodes, has been found to reliably predict the survival of patients with oral squamous cell carcinoma (OSCC), bladder carcinoma and other carcinomas with positive neck lymph nodes in recent studies (5-12). However, the association between the LND and overall survival in individuals with major salivary gland carcinoma has not yet been investigated. The present study investigated whether LND is correlated with overall survival in cases of major salivary gland carcinoma with positive lymph nodes.

\section{Patients and methods}

Population data. A total of 284 patients newly diagnosed with major salivary gland tumor at the Department of Head and Neck Surgery, Aichi Cancer Center Hospital (Nagoya, Japan), underwent tumor resection between January 2004 and May 2014; 93 of these patients were diagnosed with primary major salivary gland carcinoma on a pathological examination. Of these 93 patients, 80 received primary tumor resection with neck dissection, and 34 were diagnosed pathologically with lymph node metastasis. One patient who received preoperative chemotherapy and one patient with a past history of radiotherapy for nasopharyngeal carcinoma were excluded. Therefore, a total of 32 patients with pathologically positive lymph nodes were enrolled in the study. This study protocol was approved by the institutional review board of Aichi Cancer Center Hospital, and all patients provided their informed consent for all treatments and examinations. The anatomical locations of the primary tumor were as follows: Parotid gland, 21 patients; submandibular gland, 10 patients; and sublingual gland, 1 patient.

Tumor-node-metastasis (TNM) staging. A routine physical examination and chest radiography were performed on the first visit. The clinical stage was determined according to the findings of these examinations, as well as enhanced cervical 
computed tomography (CT) or magnetic resonance imaging. Where possible, 18F-2-fluorodeoxyglucose positron emission tomography (18F-FDG PET), or 18F-FDG PET combined with CT were performed. The findings of clinical lymph node metastasis as detected on enhanced CT, included the presence of ringed enhancement or a lymph node diameter of $\geq 10 \mathrm{~mm}$. The TNM classification criteria of the International Union Against Cancer (seventh edition) were used (13).

Pathological examination. Neck dissection, as described by the Japan Neck Dissection Study Group, was performed in an en bloc fashion (14). After carefully dividing the neck dissection samples based on the cervical region, the number of total lymph nodes was recorded. The samples and records were used in the pathological examination. In accordance with the process described in our previous report, the samples of resected tumors were fixed with formalin and embedded in paraffin (3). The pathological diagnosis (pathological $\mathrm{T}$ and $\mathrm{N}$ classification, pathological stage, histological classification, histological grade, positive surgical margin and extracapsular spread) was made by two pathologists, who compiled all reports.

Postoperative therapy. Following the pathological diagnosis, postoperative therapy was performed in patients with a positive surgical margin, extracapsular spread, multiple positive lymph nodes or high histological grade carcinoma, where possible. Following the completion of treatment, the patients were followed up at the outpatient clinic. Efforts were made to identify individuals with early locoregional recurrence and perform radical salvage therapy in such cases. The clinicopathological parameters of the patients are shown in Table I.

LND. A total of 1,346 lymph nodes were evaluated, of which $317(23.6 \%)$ were found to be pathologically positive. Based on previous studies (5-12), the LND was calculated using the following formula: LND = number of positive lymph nodes / total number of excised lymph nodes.

Statistical analysis. Statistical analysis was conducted using the JMP software package (version 9; SAS Institute, Cary, NC, USA). Correlations between LND and clinicopathological parameters (age, gender, clinical $\mathrm{T}$ and $\mathrm{N}$ classification, clinical stage, anatomical location, pathological $\mathrm{T}$ and $\mathrm{N}$ classification, pathological stage, histological classification, histological grade, positive surgical margin, extracapsular spread, positive surgical margin and/or extracapsular spread and postoperative therapy) were analyzed using the Mann-Whitney $U$ test. The survival time was defined as the period from surgery to the target event or date of last contact; target events comprised mortality for the overall survival calculation. Applying the method described in previous studies, the Kaplan-Meier technique was used to estimate survival curves, and various LND cut-off values were tested using the log-rank test in a univariate overall survival analysis $(15,16)$. Thirty-two patients were grouped into two groups based on the LND (LND $\geq 0.38$ and $<0.38$ ), as an LND of 0.38 was able to statistically distinguish the shorter from the longer survival group according to the log-rank test in the univariate survival analyses. The associations between
Table I. Clinicopathological parameters $(n=32)$.

\begin{tabular}{lr}
\hline Parameter & Value \\
\hline Age, years & \\
Mean \pm SD & $61.8 \pm 15$. \\
Gender, $\mathrm{n}$ & \\
Male & 25 \\
Female & 7 \\
Clinical T classification, $\mathrm{n}$ & \\
T1 & 0 \\
T2 & 8 \\
T3 & 7 \\
T4 & 17
\end{tabular}

Clinical $\mathrm{N}$ classification, $\mathrm{n}$

No

7

N1

4

N2

21

N3

0

Clinical stage, $\mathrm{n}$

I

II

0

2

III

IV

Anatomical location, $\mathrm{n}$

Parotid

21

Submandibular

10

Sublingual

1

Pathological T classification, $n$

T1

T2

1

T3

13

T4

18

Pathological $\mathrm{N}$ classification, $\mathrm{n}$

N0

0

N1

8

N2

N3

Pathological stage, $\mathrm{n}$

I

II

0

0

III

IV

Histological classification, $\mathrm{n}$

Adenocarcinoma, not otherwise specified $\quad 14$

Salivary duct carcinoma

Mucoepidermoid carcinoma

Adenoid cystic carcinoma

Carcinoma ex pleomorphic adenoma

Squamous cell carcinoma

Histological grade, $\mathrm{n}$

High

Others

Positive surgical margin, $\mathrm{n}$

Presence

Absence 
Table I. Continued.

\begin{tabular}{lc}
\hline Parameter & Value \\
\hline Extracapsular spread, $\mathrm{n}$ & \\
Presence & 15 \\
Absence & 17 \\
Positive surgical margin & \\
and/or extracapsular spread, $\mathrm{n}$ & 24 \\
Presence & 8 \\
Absence & \\
Postoperative therapy, $\mathrm{n}$ & 2 \\
Chemoradiation & 21 \\
Radiation & 9 \\
Absence & \\
\hline
\end{tabular}

$\mathrm{T}$, tumor; $\mathrm{N}$, node.

the two groups (LND $\geq 0.38$ and $<0.38$ ) with regard to the clinicopathological parameters (age, gender, clinical $\mathrm{T}$ and $\mathrm{N}$ classification, clinical stage, anatomical location, pathological $\mathrm{T}$ and $\mathrm{N}$ classification, pathological stage, histological classification, histological grade, positive surgical margin, extracapsular spread, positive surgical margin and/or extracapsular spread and postoperative therapy) were compared using Fisher's exact test. A Cox proportional hazards model was used for the multivariate survival analysis. Multivariate analysis was performed with adjustment for anatomical location (parotid gland/others). $\mathrm{P}<0.05$ was considered to indicate statistically significant differences.

\section{Results}

LND and clinicopathological parameters. The median LND of all patients was 0.13 (range, 0.02-0.88). The LND distribution is shown in Fig. 1, and the associations between LND and clinicopathological parameters are shown in Table II. LND was significantly correlated with pathological $\mathrm{N}$ classification $(\mathrm{P}<0.01)$, pathological stage $(\mathrm{P}=0.04)$, histological classification $(\mathrm{P}=0.02)$ and extracapsular spread $(\mathrm{P}=0.01)$.

Overall survival analysis. At the end of the study, the mean \pm SD follow-up periods among all patients, the 16 surviving patients (50.0\% vs. all) and the 16 deceased patients (50.0\%) were $23.8 \pm 19.6,25.1 \pm 19.9$ and $22.4 \pm 19.8$ months, respectively. In the entire patient group, the overall 2-, 3- and 5-year survival rates were 59.2, 43.2 and $25.9 \%$, respectively. Applying the method described previously, in our study and others $(14,15)$, various LND cut-off values were tested using the log-rank test in the overall survival analysis. A cut-off value of 0.38 for the LND had the lowest P-value in these analyses (Fig. 2) and could be used to differentiate the shorter overall survival group ( $L N D \geq 0.38, n=8$ ) from the longer overall survival group ( $\mathrm{LND}<0.38, \mathrm{n}=24$ ) based on the log-rank test, as shown in Fig. $3(\mathrm{P}=0017)$. No significant associations with clinicopathological parameters were observed in the two groups (LND $\geq 0.38$ and $<0.38$ ), as shown in Table III. A multivariate survival analysis was performed with adjustment for

Table II. Associations between LND and clinicopathological parameters $(\mathrm{n}=32)$.

\begin{tabular}{|c|c|c|c|}
\hline Parameter & $\mathrm{n}$ & $\begin{array}{c}\text { LND } \\
(\text { mean } \pm \text { SD })\end{array}$ & P-value \\
\hline Age, years & & & 0.07 \\
\hline$<65$ & 16 & $0.26 \pm 0.24$ & \\
\hline$\geq 65$ & 16 & $0.16 \pm 0.23$ & \\
\hline Gender & & & 0.73 \\
\hline Male & 25 & $0.21 \pm 0.22$ & \\
\hline Female & 7 & $0.21 \pm 0.30$ & \\
\hline Clinical T classification & & & 0.54 \\
\hline $\mathrm{T} 1-3$ & 15 & $0.24 \pm 0.27$ & \\
\hline $\mathrm{T} 4$ & 17 & $0.19 \pm 0.21$ & \\
\hline
\end{tabular}

\section{N0}

N1-2

Clinical stage

I-III

IV

Anatomical location

Parotid gland

Others

Pathological T

classification

T1-3

T4

Pathological N

classification

N1

N2

Pathological stage

III

IV

Histological classification Adenocarcinoma, NOS

Others

Histological grade

High

Others

Positive surgical margin

Presence

Absence

Extracapsular spread

Presence

Absence

Positive surgical margin and/or extracapsular spread

Presence

Absence

Postoperative therapy

Presence

Absence

${ }^{a}$ Mann-Whitney U test. LND, lymph node density; T, tumor; N, node; NOS, not otherwise specified.
$7 \quad 0.11 \pm 0.14$

$25 \quad 0.24 \pm 0.25$

$5 \quad 0.06 \pm 0.04$

$27 \quad 0.24 \pm 0.25$

$21 \quad 0.24 \pm 0.23$

$11 \quad 0.16 \pm 0.25$

0.83

$14 \quad 0.21 \pm 0.25$

$18 \quad 0.21 \pm 0.23$

$<0.01$

$8 \quad 0.03 \pm 0.01$

$24 \quad 0.27 \pm 0.25$

$<0.04$

$3 \quad 0.03 \pm 0.01$

$29 \quad 0.23 \pm 0.24$

$<0.03$

$14 \quad 0.33 \pm 0.29$

$18 \quad 0.12 \pm 0.14$

$8 \quad 0.14 \pm 0.11$

$16 \quad 0.25 \pm 0.28$

$19 \quad 0.14 \pm 0.11$

$13 \quad 0.25 \pm 0.28$

$<0.02$

$15 \quad 0.33 \pm 0.29$

$17 \quad 0.11 \pm 0.10$

0.07

$8 \quad 0.08 \pm 0.07$

$24 \quad 0.25 \pm 0.26$

0.90

$9 \quad 0.28 \pm 0.35$

$23 \quad 0.18 \pm 0.18$ 
Table III. Associations between LND $(\geq 0.38$ and $<0.38)$ and clinicopathological parameters $(n=32)$.

\begin{tabular}{lccc}
\hline & LND & LND & \\
& $\geq 0.38$ & $<0.38$ & \\
Parameter & $(\mathrm{n}=24)$ & $(\mathrm{n}=8)$ & P-value $^{\mathrm{a}}$ \\
\hline
\end{tabular}

Age, years

$<65$

$\geq 65$

Gender

Male

Female

Clinical T classification T1-3

$\mathrm{T} 4$

Clinical $\mathrm{N}$ classification

N0

N1-2

Clinical stage

I-III

IV

Anatomical location

Parotid gland

Others

Pathological T classification

T1-3

$\mathrm{T} 4$

Pathological $\mathrm{N}$ classification

$$
\text { N1 }
$$

N2

Pathological stage

III

IV

Histological classification

Adenocarcinoma, NOS

Other

Histological grade

High

Other

Positive surgical margin

Presence

Absence

Extracapsular spread

Presence

Absence

Positive surgical margin

and/or extracapsular spread

Presence

Absence

$16 \quad 8$

80

Postoperative therapy

Presence

Absence

0.22

$10 \quad 6$

142

0.65

18

$$
6
$$

$10 \quad 5$

$14 \quad 3$

$\begin{array}{ll}6 & 1\end{array}$

18

$$
5
$$

0

8

10

14

0.08

80

168

$$
3
$$

86

162

$10 \quad 1$

$14 \quad 7$

$\begin{array}{ll}13 & 6 \\ 11 & 2\end{array}$

96

6

2

${ }^{a}$ Fisher's exact test. LND, lymph node density; T, tumor; N, node; NOS, not otherwise specified.

0.08
Table IV. Multivariate survival analysis ${ }^{\mathrm{a}}$.

\begin{tabular}{lccc}
\hline & \multicolumn{3}{c}{ Overall survival } \\
\cline { 2 - 4 } Parameter & HR & $95 \%$ CI & P-value \\
\hline $\begin{array}{l}\text { LND }(\geq 0.38 /<0.38) \\
\begin{array}{l}\text { Anatomical location } \\
\text { (parotid/others) }\end{array}\end{array}$ & 4.02 & $1.21-13.43$ & $\mathrm{P}<0.03$ \\
\hline
\end{tabular}

${ }^{\mathrm{a}}$ Cox proportional hazards model. LND, lymph node density; HR, hazard ratio; CI, confidence interval.

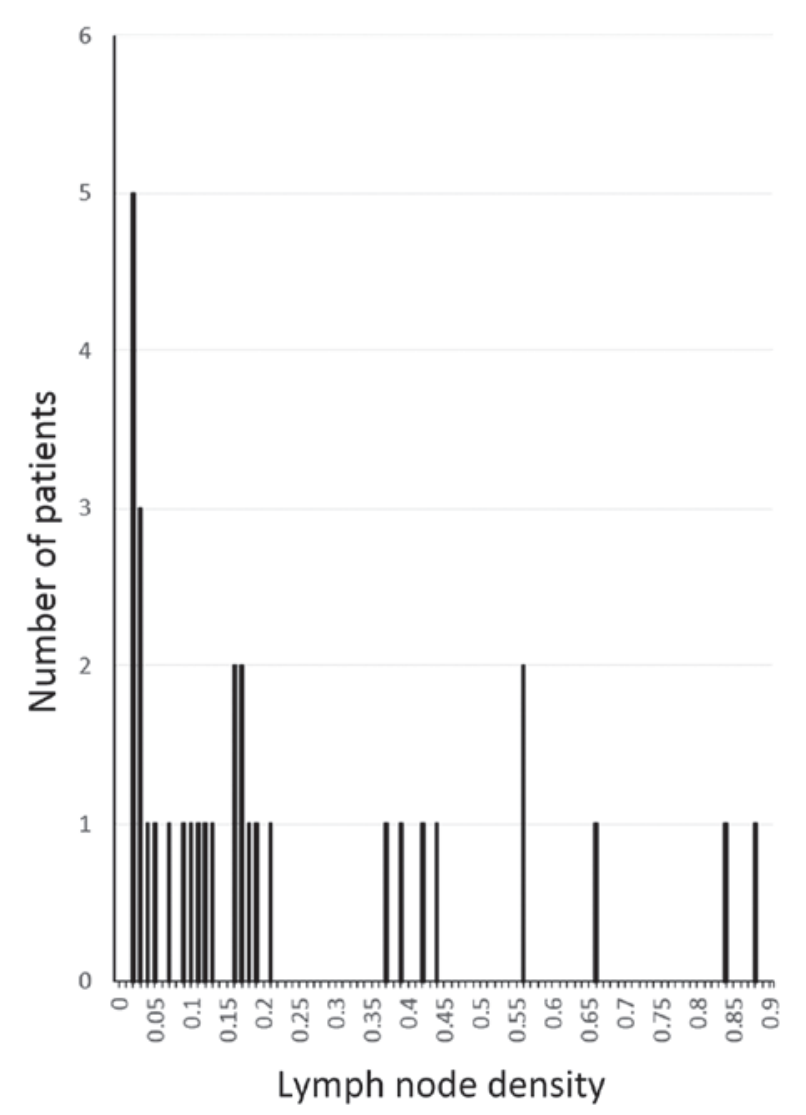

Figure 1. Lymph node density and number of patients.

anatomical location (parotid gland/others) for overall survival. Consequently, an LND of $\geq 0.38$ was confirmed to be associated with a significantly shorter survival time. The results of the multivariate analysis for survival are presented in Table IV.

\section{Discussion}

The results of the current study demonstrated, for the first time, that an LND of $\geq 0.38$ in patients with major salivary gland carcinoma exhibiting pathological lymph node metastasis is significantly associated with a shorter overall survival time.

The LND determined by pathological examination has emerged as a prognostic parameter for various types of cancer, including bladder, esophageal and oral cancers (5-12). The ratio of the LND weights three factors that may influence nodal staging: Tumor (the true number of positive 


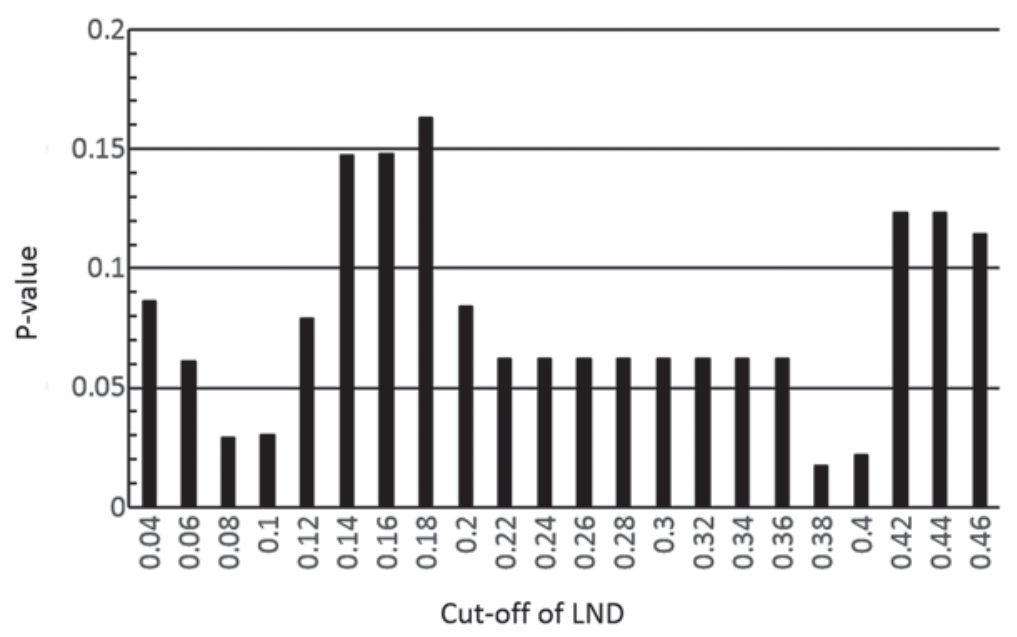

Figure 2. P-values according to the log-rank test for overall survival using different cut-off values for the LND in 32 major salivary gland carcinoma patients with positive lymph nodes. LND, lymph node density.

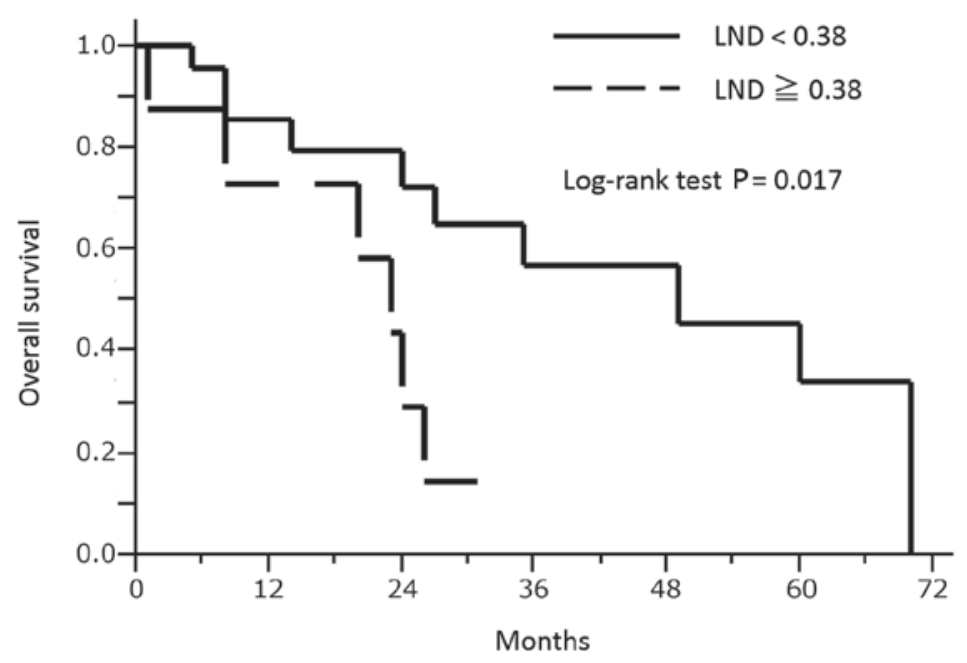

Figure 3. Associations between LND and survival in 32 major salivary gland carcinoma patients with positive lymph nodes (Kaplan-Meier method). An LND of $\geq 0.38$ was found to be associated with a significantly shorter overall survival time. The log-rank test was used for the statistical analysis. LND, lymph node density.

lymph nodes), surgical (the actual number of nodes removed during neck dissection) and sampling (the completeness of the pathological analysis) factors (5). Recently, a multi-institutional international study group comprising 11 cancer centers across the globe revealed the LND to be a significant predictor of overall survival time among 4,254 patients with OSCC (5). Furthermore, a number of studies have reported that LND may be used to predict the survival time of OSCC patients treated with different neck dissection procedures, such as unilateral or bilateral neck dissections (5-10). The present findings, demonstrating an association between LND and overall survival rate, are in concordance with this previous evidence (5-10).

With regard to head and neck cancer, numerous studies have reported an association between LND and overall survival in patients with OSCC (5-9). Furthermore, Rudra et al (10) reported that LND predicts overall survival time in subjects with lesions in various sites of the head and neck, including the oropharynx, oral cavity and larynx/hypopharynx; however, this study did not investigate the relationship between LND and overall survival in patients with major salivary gland carcinoma (10). In the present study, the association between LND and overall survival was assessed in subjects with major salivary gland carcinoma, demonstrating that an LND of $\geq 0.38$ in patients with pathological lymph node metastasis is significantly associated with a shorter overall survival time.

The predominant limitation of the current study was the relatively small number of subjects in the sample. Hence, future analyses of large numbers of patients will yield more statistically accurate results, with greater potential for application of the findings.

In conclusion, the present study demonstrated, for the first time, that an LND of $\geq 0.38$ in patients with major salivary gland carcinoma exhibiting pathological lymph node metastasis is significantly associated with a shorter overall survival time. Therefore, LND is a prognostic factor in individuals with major salivary gland carcinoma. 


\section{Acknowledgements}

The current study was supported by Japan Society for the Promotion of Science Grants-in-Aid for Scientific Research (grant no. 24791821). Part of this study was presented at the 2014 World Cancer Congress, December 3-6, 2014, Melbourne, Australia (17).

\section{References}

1. Ettl T, Schwarz-Furlan S, Gosau M and Reichert TE: Salivary gland carcinomas. Oral Maxillofac Surg 16: 267-283, 2012.

2. Barnes L, Eveson JW, Reichart P and Sidransky D (Eds): World Health Organization classification of tumours Pathology and genetics of head and neck tumours. IARC Press, Lyon, 2005.

3. Okabe M, Miyabe S, Nagatsuka H, Terada A, Hanai N, Yokoi M, Shimozato K, Eimoto T, Nakamura S, Nagai N, et al: MECT1-MAML2 fusion transcript defines a favorable subset of mucoepidermoid carcinoma. Clin Cancer Res 12: 3902-3907, 2006.

4. Amit M, Na'ara S, Sharma K, Ramer N, Ramer I, Agbetoba A, Glick J, Yang X, Lei D, Bjoerndal K, et al: Elective neck dissection in patients with head and neck adenoid cystic carcinoma: An international collaborative study. Ann Surg Oncol 22: 1353-1359, 2015

5. Patel SG, Amit M, Yen TC, Liao CT, Chaturvedi P, Agarwal JP, Kowalski LP, Ebrahimi A, Clark JR, Cernea CR, et al: Lymph node density in oral cavity cancer: Results of the International Consortium for Outcomes Research. Br J Cancer 109: 2087-2095, 2013.

6. Kim SY, Nam SY, Choi SH, Cho KJ and Roh JL: Prognostic value of lymph node density in node-positive patients with oral squamous cell carcinoma. Ann Surg Oncol 18: 2310-2317, 2011.

7. Gil Z, Carlson DL, Boyle JO, Kraus DH, Shah JP, Shaha AR, Singh B, Wong RJ and Patel SG: Lymph node density is a significant predictor of outcome in patients with oral cancer. Cancer 115: 5700-5710, 2009.

8. Amar A, Rapoprt A, Curioni OA, Dedivitis RA, Cernea CR and Brandáo LG: The density of metastatic lymph node as prognostic factor in squamous cell carcinoma of the tongue and floor of the mouth. Braz J Otorhinolaryngol 78: 86-90, 2012 (In Portuguese).
9. Liao CT, Hsueh C, Lee LY, Lin CY, Fan KH, Wang HM, Huang SF, Chen IH, Kang CJ, Ng SH, et al: Neck dissection field and lymph node density predict prognosis in patients with oral cavity cancer and pathological node metastases treated with adjuvant therapy. Oral Oncol 48: 329-336, 2012.

10. Rudra S, Spiotto MT, Witt ME, Blair EA, Stenson K and Haraf DJ: Lymph node density - prognostic value in head and neck cancer. Head Neck 36: 266-272, 2014.

11. Kassouf W, Leibovici D, Munsell MF, Dinney CP, Grossman HB and Kamat AM: Evaluation of the relevance of lymph node density in a contemporary series of patients undergoing radical cystectomy. J Urol 176: 53-57, 2006.

12. Ooki A, Yamashita K, Kobayashi N, Katada N, Sakuramoto S, Kikuchi S and Watanabe M: Lymph node metastasis density and growth pattern as independent prognostic factors in advanced esophageal squamous cell carcinoma. World J Surg 31: 2184-2191, 2007.

13. Sobin LH, Wittekind C and Gospodarowicz M: International Union Against Cancer TNM classification of malignant tumours. 7th edition. Wiley-Blackwell, New York, NY, USA, 2009.

14. Hasegawa Y and Saikawa M: Update on the classification and nomenclature system for neck dissection: Revisions proposed by the Japan Neck Dissection Study Group. Int J Clin Oncol 15: $5-12,2010$.

15. Van Baardwijk A, Dooms C, van Suylen RJ, Verbeken E, Hochstenbag M, Dehing-Oberije C, Rupa D, Pastorekova S, Stroobants S, Buell U, et al: The maximum uptake of (18)F-deoxyglucose on positron emission tomography scan correlates with survival, hypoxia inducible factor-1alpha and GLUT-1 in non-small cell lung cancer. Eur J Cancer 43: 1392-1398, 2007.

16. Suzuki H, Kato K, Fujimoto Y, Itoh Y, Hiramatsu M, Naganawa S, Hasegawa Y and Nakashima T: Prognostic value of (18) F-fluorodeoxyglucose uptake before treatment for pharyngeal cancer. Ann Nucl Med 28: 356-362, 2014.

17. Suzuki H: Lymph node density is a prognostic factor in patients with major salivary gland carcinoma. Asia-Pacific Journal of Clinical Oncology 10: 1-264, 2014 